\title{
La acción vecinal más allá del barrio: el caso del distrito Nou Barris en Barcelona
}

\section{The neighborhood action beyond the neighborhood: The case of Nou Barris district in Barcelona}

\section{Francisco Letelier}

Universidad Católica del Maule - CEUT, Talca, Chile.

fcoletelier@gmail.com

\section{Fabiana Valdosky}

Grupo de Geografía Crítica Radical, Universidade de São Paulo, São Paulo, Brasil.

\section{Resumen}

La concepción neoecológica de barrio fue adoptada en los años 1980 por diversos gobiernos de Europa en el marco de la agenda neoliberal. Asumida de manera acrítica como escala privilegiada (si no única) para la gobernanza urbana, la idea de barrio ha contribuido a legitimar e institucionalizar una acción vecinal fragmentada y despolitizada, con escasa capacidad de producir lo urbano. Desde una posición crítica a esta noción de barrio y su implícita manera de concebir las relaciones entre las comunidades y las políticas urbanas, este artículo propone una aproximación multiescalar y política de lo vecinal, resumida en el concepto de geografías de la acción vecinal. Para ilustrar esta aproximación se analiza el caso del distrito de Nou Barris, en Barcelona, donde la actuación de las organizaciones traspasa los límites de la noción hegemónica de barrio, constituyendo relaciones más complejas y territorialidades más amplias que buscan incidir en lo urbano. Se concluye que la acción vecinal puede adoptar diversas 'geografías' y constituir nuevas configuraciones relacionales no constreñidas por espacialidades limitantes. Puede escalar sus ámbitos de actuación desde la proximidad residencial inmediata a la ciudad entera, y desempeñar un rol fundamental al intermediar entre el habitar cotidiano y el sistema político.

Palabras clave: Barrio, urbanismo neoliberal, redes vecinales, geografías de la acción vecinal

\begin{abstract}
The neoecological conception of the neighborhood was adopted in the 1980s by various governments in Europe using the framework of the neoliberal agenda. Assumed in an uncritical way as a privileged (if not unique) scale for urban governance, the idea of the neighborhood has both legitimized and institutionalized a fragmented and depoliticized neighborhood action, with little capacity to produce an urban context. From a critical position to this notion of neighborhood and its implicit way of conceiving relations between communities and urban policies, this article proposes a multiscale and political approach of the neighborhood, by applying the concept of geographies of neighborhood action. To illustrate this approach, the case of the district of Nou Barris, in Barcelona, is analyzed, where the actions of the organizations go beyond the limits of the hegemonic notion of the neighborhood, constituting more complex relationships and broader territorialities that seek to influence the urban. It is concluded that the neighborhood action can adopt different 'geographies' and constitute new relational configurations not constrained by limiting spatialities. It can scale its fields of action from the immediate residential proximity to the entire city, and play a key role in intermediating between the everyday life and the political system.
\end{abstract}

Keywords: geographies of neighborhood action, neighborhood, neighborhood networks, neoliberal urbanism.

Documento recibido el 14 de junio de 2019 y aceptado el 19 de diciembre de 2019.

Este artículo ha sido elaborado en el contexto de una beca de doctorado del programa de capital humano avanzado del Consejo Nacional de Ciencia y Tecnología de Chile.

Cómo citar: Letelier, F. y Valdosky, F. (2019). La acción vecinal más allá del barrio: el caso del distrito Nou Barris en Barcelona. Revista de Urbanismo, 40, 1-16. https://doi.org/10.5354/0717-5051.2019.53618 


\section{Introducción}

El barrio vivido, el lugar del encuentro cotidiano, de relaciones de ayuda mutua a la vez que de conflicto; de refugio y resistencia a la vez que de control, ha venido debilitándose en su capacidad de producir la ciudad. En su lugar se ha impuesto un barrio concebido y abstracto, objeto de la planificación y del urbanismo. Se trata de un espacio diagramado y delimitado que segmenta y segrega la ciudad, y la prepara para ser intervenida y consumida no como totalidad, sino como una sumatoria de partes.

El fundamento conceptual del uso ideológico de la idea de barrio es la concepción neoecológica de lo vecinal. Originada en los trabajos de la Escuela de Chicago a inicios de 1900 (Park, Burgess, \& McKenzie, 1925), tal aproximación fue pilar del modelo de planificación e intervención urbana en Estados Unidos y Europa durante al menos cuarenta años. Desde esta perspectiva, las unidades urbanas que forman los barrios se constituyen y reproducen naturalmente a partir de dinámicas ecológicas de cooperación interna y competencia con el entorno, lo que les otorga límites muy claros. Se presume que lo urbano no sería un continuo, sino que estaría conformado por múltiples fragmentos que se tocan, pero no se interpenetran (Suttles, 1972); y en este sentido, se los asume como espacios sin conexión con la totalidad, y tampoco con los procesos políticos y económicos que los producen.

Esa misma concepción es la que fue implementada en los años 1980 por diversos gobiernos de Europa, en el marco de la agenda neoliberal y sus políticas de reescalamiento (Brenner, 2004; Madden, 2014). Las iniciativas a las que así se dio origen estaban destinadas a hacer frente a los efectos de las crecientes desigualdades y violencias urbanas producidas por los agresivos procesos de destrucción creativa, agravados por un capitalismo financiero volcado en inversiones urbanas que producían procesos sistemáticos de valorización de ciertas áreas y precarización de otras. Colocar el barrio en el centro de las políticas urbanas cumplía un doble propósito: por un lado, movilizar a la propia comunidad en la solución de sus problemas (Harvey, 1997); y por otro, contener los problemas en espacios acotados, buscando que su solución se originara en el lugar mismo donde "se producía" (Harvey, 1997; Tapia, 2018) y evitando la crítica al modelo de desarrollo sociourbano en su conjunto. Esta estrategia ha convertido la escala de barrio en el lugar privilegiado para ensayar políticas urbanas (Martin, 2003; Silver, 1985; Wellman \& Leighton, 1979), distribuir servicios, intervenir en función de los objetivos de la regeneración y promover procesos de inclusión y cohesión social (Atkinson, Dowling, \& McGuirk, 2009).

La consolidación de la escala barrial en las políticas urbanas ha monopolizado el modo de entender la acción vecinal. En primer lugar, la ha acotado espacialmente, concibiéndola como desconectada de la totalidad urbana. En esta línea, ha condicionado su existencia a vínculos afectivos-cohesivos, organizándola y adecuándola a los programas urbanos oficiales. Y como consecuencia de lo anterior, la ha entendido restringida a geografías y territorialidades que carecen de la influencia necesaria para participar de manera incidente en la producción de lo urbano (Letelier, 2018; Tapia, 2018).

Discutiendo la idea dominante de barrio a partir de aproximaciones que ponen acento en la dimensión relacional del espacio; en la configuración reticular de lo vecinal y en su multiescalairidad, se plantea la hipótesis de que la acción vecinal puede no estar referida solo a un espacio delimitado, sino al ámbito de relaciones que se establecen en el marco del habitar (Keller, 1979; Massey, 2012). Al no estar este marco determinado por restricciones espacio-temporales fijas, la acción vecinal debe entenderse como un flujo de relaciones complejas, abiertas y dinámicas, articuladas en diversas geografías (Massey, 2012; Merrifield, 2011).

A partir de esta conceptualización y utilizando diversas fuentes de información, el artículo tiene por objetivo analizar el caso del distrito de Nou Barris. En torno a él nos preguntamos: ¿de qué manera la acción vecinal en Nou Barris discute la concepción dominante de barrio? Los resultados muestran que la acción vecinal traspasa los límites de la noción de barrio y escala hacia arriba, al 'distrito' y a la ciudad, construyendo nuevas geografías y territorialidades. Se concluye que la acción vecinal aumenta su superficie de contacto con los diferentes niveles de complejidad que participan en la producción de lo urbano; al mismo tiempo se politiza, en la medida en que las problemáticas de las cuales se hace cargo se vinculan con la idea de injustica y se redefinen sus agendas y estrategias. 
El artículo se divide en cuatro partes. En la primera, se ubica el barrio en la agenda urbana neoliberal como una territorialidad específica, y se señala su papel como "geografía de contención". En la segunda se esboza un marco de comprensión de las relaciones vecinales que las sitúa más allá del "barrio", perspectiva que las vincula a geografías y territorialidades múltiples. En la tercera parte se revisa el caso de Nou Barris en Barcelona, España; se reconstruyen las trayectorias de asociatividad y acción vecinal que allí han tenido lugar, y su relación con las políticas urbanas y territoriales; y se discuten los resultados de tal enfoque a la luz de la noción de geografía de la acción vecinal. Finalmente, en la cuarta parte se concluye que la acción vecinal debe entenderse dentro de procesos de constitución de nuevas configuraciones relacionales. No estaría constreñida por espacios predefinidos, sino que ella misma crearía sus propias espacialidades y geografías. Como consecuencia, podría escalar, reescalar y desescalar sus ámbitos de actuación desde la proximidad residencial inmediata, a la ciudad entera y más allá.

\section{La idea de barrio en la agenda neoliberal}

Desde la visión neoecológica del barrio (Park et al., 1925) y en sus aplicaciones prácticas como 'Unidad Vecinal' (Perry, 1929), las unidades urbanas que forman los vecindarios se constituyen y reproducen naturalmente a partir de dinámicas ecológicas de cooperación interna y competencia con el entorno, lo que les otorga límites muy claros: "cada formación u organización ecológica sirve como una fuerza selectiva o magnética que atrae a los elementos de población apropiados y repele las unidades incongruentes" (Park et al., 1925, pp. 77-78). Según ya señalado, lo urbano no sería un continuo, sino que estaría conformado por múltiples fragmentos contiguos, pero que no se penetran recíprocamente (Suttles, 1972).

De acuerdo con Park y sus colegas de la Escuela de Chicago (1925), los vecindarios, concebidos por ellos como formando parte de un orden natural, son portadores de valores que aseguran la socialización cohesiva de sus miembros y constituyen un refugio frente a la amenaza de una ciudad cada vez más impersonal. Tal sería la vía por la que se resguarda, promueve y restituye la comunidad vecinal (Bettin, 1982; Martínez, 1999). Desde esta perspectiva, tal restitución se hace en un contexto en el cual la configuración social y la organización de la ciudad, y sus resultados, se entienden como procesos naturales, que escapan a toda responsabilidad social. Bajo esta óptica se legitima, como posible y deseable, actuar sobre cada vecindario como una realidad independiente, sin tener que incorporar en el proceso las condiciones estructurales que lo rodean y producen.

A partir de la década de 1980 y de la mano de concepciones neoliberales que reivindicaron lo comunitario como espacio liberado de la coacción estatal, la idea neoecológica de barrio se ha venido instalando como la concepción dominante (Madden, 2014). Esto ha sido facilitado por el enorme poder de la idea de barrio para producir mapas cognitivos que clasifican, separan y simplifican lo urbano (Suttles, 1972). Un poder cuya efectividad descansa en su naturaleza ideológica y en su capacidad para encarnar el sentido común a partir del cual entendemos las relaciones urbanas (Lefebvre, 2013). Como señala Jane Jacobs (2011), 'barrio' es "una palabra que ha llegado a sonar como un poema de San Valentín" (p. 143).

El barrio ha sido presentado como la escala de gobernanza urbana ideal para lo que, a nivel global, se ha denominado el "nuevo localismo" (Brenner \& Theodore, 2002): la búsqueda de soluciones a los problemas sociales y económicos mediante el traspaso de la responsabilidad a las áreas locales (Martin, 2003). Esto implica considerar que los problemas son del barrio y que, por tanto, deben solucionarse en él. En otra dirección, Garnier (2011) señala que la descentralización que supuestamente conllevaría esta escala de barrio es ficticia, ya que es operada por los órganos del Estado, sin que los interesados tengan realmente la posibilidad de expresarse al respecto. Tras este interés por los barrios existiría, de hecho, una agenda neoliberal cuyos propósitos terminan convirtiendo la ciudad en un sustrato para la obtención de plusvalías vía creciente gentrificación, segregación y sobreproducción de espacios urbanos exclusivos (Davidson, 2008; Harvey, 1997).

En los países occidentales, la concepción dominante de barrio se ha venido consolidando a través de múltiples programas públicos (Atkinson, Dowling, \& McGuirk, 2009). En el ámbito europeo, el despliegue de políticas barriales se relaciona con tensiones raciales, desigualdad, segmentación social, falta de cohesión social y 
fragmentación del paisaje urbano, problemas que caracterizan los denominados "barrios en crisis" (quartiers en crisis) (Andersson \& Musterd, 2005). Los programas de escala barrial intervienen focalizadamente: definen áreas delimitadas en función de las carencias de infraestructura, servicios y residencia de la población más pobre, y evitan la discusión de las lógicas urbanas que causan los problemas. En esta perspectiva, se asume que el problema está "en el barrio", no en su inserción en un sistema económico desigual, ni vinculado a su relación con las políticas o gobernanza urbana.

Como corolario, es el barrio el que debería cambiar, y para eso se requiere de habitantes que confíen unos en otros y participen en el proceso de mejoramiento; es decir, capaces de movilizar capital social. Es así que, al pensar el barrio, no se concibe al ciudadano-habitante en su derecho a producir el territorio desde una reflexión crítica en torno a su rol en la sociedad y su relación con el Estado y el mercado, capaz de definir horizontes políticos más amplios para su acción (Letelier, 2018; Tapia, 2018).

Puesta en el marco de la discusión acerca de la naturaleza del espacio, la visión dominante de barrio se ubicaría en lo que se denomina 'espacio absoluto': un espacio contenedor, fijo, que actúa sobre todos los objetos que contiene sin que ellos puedan ejercer acciones recíprocas sobre él (Harvey, 2012; Massey, 2007). Como espacio absoluto, el barrio limita la forma y el alcance de la acción y relaciones vecinales. Acota su espacialidad en torno a una lógica de proximidad restringida, anclada en lo residencial, y da primacía a los vínculos fuertes en desmedro de los vínculos débiles (Wellman, 1979; 2001). El ideal de comunidad niega la diferencia que se presenta bajo la forma del distanciamiento temporal y espacial que caracteriza los procesos sociales (Young, 2000, p. 380). La "comunidad", contenido del barrio, queda consagrada como único código de trabajo para la cohesión (Suttles, 1972; Wellman, 2001).

Se tiende a pensar que el espacio es poseedor de una vida independiente de las fuerzas, instituciones y políticas que lo conforman (Lefebvre, 1991; Madden, 2014). En este marco, los sujetos restringirían sus agendas a la reproducción cotidiana de las comunidades, desconectándose de las causas estructurales de los problemas del barrio y reduciendo el sentido del habitar, sus agendas y estrategias. La concepción de barrio como espacio absoluto se expresa y reproduce a través de geografías de relaciones urbanas contenidas, que restringen la capacidad de los sujetos para crear el espacio. Dicho de otro modo, en el marco del barrio así concebido, la acción vecinal se desconecta de su potencial de transformación y producción de lo urbano. Cada comunidad actúa en su 'metro cuadrado' y tiene expectativas de éxito acotadas, puestas fundamentalmente en capturar recursos que no requieran una presión mayor y que mantienen a los Estados y gobiernos en su espacio de confort. Tal fragmentación del espacio -y de la acción - impide a los actores concebir las potencialidades de la acción conjunta, o la multiplicidad de recursos combinados que pudieran ser movilizados (Letelier, 2018; Letelier, Tapia, \& Boyco, 2018).

\section{La acción vecinal más allá de la idea barrio}

En una dirección opuesta a la aproximación neoliberal a la idea de barrio, diversos autores han conceptualizado el ámbito vecinal como primordialmente relacional, no restringido por espacialidades programadas o abstractas. Estas relaciones construyen redes que conectan unas comunidades y organizaciones con otras más allá de las delimitaciones administrativas, y les permiten actuar en diversas escalas territoriales, desde la calle a la ciudad entera. A continuación, exploraremos cómo estas formulaciones permiten proponer la idea de 'geografías de la acción vecinal'.

El ámbito vecinal como espacio relacional. A fines de los años 1970, Susane Keller (1979) concluyó que lo más propio de los vecindarios no es su delimitación física ni su forma urbana, sino las relaciones y prácticas de vecindad. Lo vecinal sería ante todo expresión de lo que hacen los vecinos en tanto tales, las relaciones y redes que construyen (Rodríguez, 2008; Wellman, 2001). Así, el espacio (vecinal) solo existiría en función de las relaciones que se desarrollan en él (Harvey, 2012) y se construye (y transforma) en virtud de los vínculos, redes y flujos que establece con distintas escalas (Massey, 2012). El ámbito vecinal, por lo tanto, sería el espacio social producido y transformado continuamente por relaciones que se tejen en torno al habitar. Haciendo una analogía con la distinción que Neil Brenner (2017), tomando una idea de Lefebvre, hace entre ciudad y 'lo urbano', el barrio sería el 
objeto que se da por descontado y que se utiliza irreflexivamente para describir la vida social urbana, mientras que 'lo vecinal' sería el proceso, lo que realmente se debe observar.

Concebir lo vecinal como relacional implica entender que su contenido es definido por las propias relaciones, los usos, las necesidades y las estrategias de los sujetos (Keller, 1979; Suttles, 1972). En esta perspectiva, el contenido de lo vecinal se construye en la práctica social; así, lo que ayer fue considerado propio de lo vecinal, no lo es hoy; como espacio vivido, es un espacio de posibilidades, abierto a la creatividad de los actores (Lefebvre, 1969; Merrifield, 2011; Purcell, 2014).

Desde esta perspectiva, lo vecinal debe ser observado considerando también el influjo de los procesos globalizadores, pero sin obviar el papel de los lugares. Si bien en el ámbito de lo vecinal se han venido produciendo procesos de desanclaje - relaciones sociales que se despegan de sus contextos locales de interacción y se reestructuran en intervalos espacio-temporales indefinidos (Giddens, 1993) - , el resultado no es que las personas dejan de llevar sus vidas en las localidades reales. Lo que ocurre es que al debilitarse sus constreñimientos espaciales, las relaciones de cotidianeidad y proximidad se complejizan (Villasante, 1999). Así, según señala Massey (2012), en el escenario actual, tanto la dimensión local como la global tienen efectos sobre la manera en que se construye el orden social de un vecindario. Por tal razón, en vez de pensar los lugares como áreas contenidas dentro de unos límites (barrios), podemos imaginarlos como momentos articulados en redes de relaciones e interpretaciones sociales construidas, en buena parte, a una escala mucho mayor.

De lo anterior se concluye que las relaciones vecinales y la acción vecinal pueden estar referenciadas por experiencias urbanas de diversas intensidades y formas de proximidad. Es posible identificar al menos cuatro ámbitos de referencia para las relaciones vecinales. El primero es el de la sociabilidad cotidiana, del vínculo cara a cara, basado tanto en la proximidad residencial como en la reiteración del encuentro. El segundo es el acceso, uso y/o exclusión de bienes públicos, infraestructuras y equipamientos de la ciudad: transporte, educación, salud, espacio público recreativo, etc. El tercero es el de los conflictos urbanos, amenazas contra las que las personas se articulan en determinados espacios y tiempos. El cuarto es el ámbito de las desigualdades, entendidas en su dimensión espacial, y la lucha por la distribución del bienestar en la ciudad y, siguiendo a Merrifield (2011), también en lo global: el empleo, la migración, la educación, las pensiones. Las relaciones vecinales pueden estar organizadas en uno o en muchos de estos niveles al mismo tiempo, y cada uno de ellos implica espacialidades, formas de organización y agendas distintas y potencialmente complementarias.

Lo vecinal red. Para la concepción dominante de "barrio", en las relaciones vecinales predominan los vínculos de lazo fuerte (Leighton, 1979; Wellman, 1979; Wellman \& Young, 2000), las comunidades contenidas, insertas en espacios urbanos delimitados y sin contacto con comunidades de zonas distintas. Pero - como se apuntó en el apartado anterior-, las relaciones vecinales no están constreñidas por lo residencial, que sería el lugar privilegiado de los vínculos de lazo fuerte, sino que pueden establecerse obedeciendo a distintos grados de proximidad y en orden a las nuevas dinámicas localglobales. Considerar lo vecinal en una dialéctica de proximidades-distancias lleva a tener que introducir la noción de vínculo de lazo débil (Granovetter, 1973). Tales vínculos actúan como puentes para conectar grupos y dar lugar a estructuras más grandes y complejas (Espinoza, 1998, 2003; Granovetter, 1973). La imagen de lo vecinal que emerge se opone a la idea de las comunidades como constituidas exclusivamente por relaciones de reciprocidad y solidaridad (Panfichi, 1996).

Aunque suele asociarse la idea de cohesión con la de vínculos fuertes, la sola existencia de vinculaciones estrechas produce estructuras frágiles -es decir, poco cohesionadas -, pues homogeneízan a los integrantes de los grupos, reduciendo la capacidad de vincularse entre círculos sociales distantes. Este tipo de configuración de lazos tendrá dificultades estructurales para desplegar estrategias comunes de mayor alcance (Granovetter, 1973). Una perspectiva cohesiva que incorpora los vínculos de lazo débil tiene un carácter más estructural, donde es la caracterización del vínculo lo que nos permite acercarnos a saber si un grupo social es o no cohesionado.

Al observar lo vecinal desde este enfoque, emerge una imagen mucho más compleja: 'comunidades' integradas 
por pequeñas redes de lazos fuertes que se superponen entre sí. En esta imagen, los lazos débiles actúan como puentes entre grupos de lazos fuertes (Panfichi, 1996). Lo vecinal se entiende como una red: un conjunto de actores -individuos, grupos, organizaciones y/o comunidadesvinculados unos a otros a través de una o un conjunto de relaciones sociales (Wellman, 1979). Estas relaciones están basadas en diferentes patrones de lazos (y niveles y tipos de proximidad), los cuales pueden trascender los límites físicos de un área encapsulada (Sanz, 2003). Así, en una determinada zona de la ciudad pueden coexistir varios grupos, cada uno articulado internamente por lazos fuertes (las organizaciones de un barrio), articulados a su vez entre ellos por lazos débiles y, al mismo tiempo, articulada esta red con otras redes: una red de redes.

\section{Escalas de acción vecinal}

Denominaremos 'geografía de la acción vecinal' a la espacialidad y la estructura que adoptan las articulaciones y actuaciones de las organizaciones y colectivos vecinales en un momento determinado. Esta geografía condicionará las agendas y estrategias de los actores sociales; es decir, su ejercicio de territorialidad, asumido como la puesta en escena del poder por parte de colectivos urbanos en relación con un espacio (Lopes de Souza, 2016; Raffestin \& Butler, 2012). Así, por ejemplo, la geografía promovida por la idea dominante de barrio acota la espacialidad de la acción vecinal a polígonos bien delimitados y a formas tradicionales de organización y vinculación con el Estado. Esto, a su vez, limita la capacidad de las organizaciones para asumir agendas políticas que problematicen la relación entre lo que ocurre en el barrio y los contextos urbanos y sociales más amplios.

La capacidad de las relaciones vecinales para construir territorialidades en diversas escalas ya fue observada hace décadas por Jane Jacobs (2011 [1961]) y Gerard Suttles (1972). Para ambos. la acción vecinal puede tener como escenario una calle, el área de residencia inmediata, la localidad de algunas cuadras o toda la ciudad. Esta perspectiva aparece nuevamente en la forma en que Neil Brenner entiende la escala, no como una realidad objetiva y estática, sino como un medio de lucha política por el control del espacio y del proceso de acumulación (Sevilla, 2017). La escala de lo vecinal estará en relación con los propósitos y las agendas que las organizaciones vecinales se planteen (Madden, 2014; Suttles, 1972).

Jacobs (2011, p. 159) sostiene que el vecindario residencial, lo que generalmente se denomina barrio, sería demasiado grande para poseer capacidades eficaces o significado real en tanto que habitar urbano cotidiano; y, al mismo tiempo, sería demasiado pequeño para funcionar como un área capaz de construir poder. En cambio, apuesta por el distrito y lo entiende como lugar de articulación política del habitar cotidiano, cuya principal función sería mediar entre los barrios, desamparados políticamente, y la poderosa ciudad en su conjunto. Los distritos serían así traductores de las experiencias de la vida real en políticas y objetivos de la ciudad.

Jacobs no entiende el distrito como una delimitación político-administrativa o una mera sumatoria de organizaciones, sino como una unidad integral de poder y opinión lo bastante grande para ser tenida en cuenta. En los distritos según los concibe Jacob, la acción vecinal es capaz de articular la vida cotidiana (y sus problemas) con la esfera de las decisiones urbanas. Son una geografía de la acción vecinal que conjuga y vincula distintas espacialidades del habitar a través de estructuras de organización flexibles.

\section{Metodología}

La investigación utiliza como metodología el estudio de caso. En un primer momento se describe para el caso español, y de Barcelona en particular, la trayectoria histórica y político-institucional de lo vecinal y la introducción de la escala de barrio en las políticas urbanas y sociales durante los últimos cuarenta años.

En un segundo momento se observa el caso de Nou Barris. En primer término, se describe brevemente las características socioeconómicas y demográficas del distrito, y luego se reconstruyen sus trayectorias vecinales, tomando un horizonte de cincuenta años y poniendo énfasis en tres dimensiones de lo vecinal: las prácticas de articulación y sus espacialidades; las agendas y objetivos; y la acción vecinal y su relación con el sistema político.

Para informar el caso se han utilizado diversas fuentes. En la reconstrucción de la trayectoria de las políticas urbanas y vecinales y la introducción de la noción de barrio 
en España y Barcelona, se ha recurrido a la revisión bibliográfica y documental. La reconstrucción de las trayectorias de articulación de Nou Barris se ha sustentado en distintos instrumentos: i) entrevistas a líderes (hombres y mujeres) de asociaciones y organizaciones vecinales de Nou Barris realizadas entre 2014 y 2019 por los propios investigadores, complementadas con otras disponibles en trabajos académicos; ii) documentos de trabajo de las propias organizaciones vecinales, tales como diagnósticos participativos, planes y propuestas de desarrollo; iii) material periodístico y notas de prensa de medios escritos; y iv) un conjunto de artículos y trabajos académicos dedicados al caso Nou Barris.

\section{Las geografías de la acción vecinal en Nou Barris}

La introducción de la idea de barrio y la institucionalización de la acción vecinal en España y Barcelona. Durante la dictadura franquista, el movimiento vecinal constituyó un espacio donde se articularon diversas organizaciones sociales y políticas, y uno de los ámbitos activos de resistencia, junto al sindical y al político en la clandestinidad. Fue activo en la defensa de los derechos civiles, y tuvo importantes logros en el plano del derecho a la vivienda y en la lucha por evitar grandes proyectos especulativos (Mesa, 2017). El movimiento vecinal fue en esos años un actor político de primera línea, con capacidad de pensar y actuar en la ciudad (Borja, 1975, p. 99).

Uno de los aspectos más destacables del movimiento vecinal fue su capacidad de articularse en redes de asociaciones para abordar problemas que iban más allá del propio barrio. La lógica de escalar las luchas urbanas está muy en relación con el influjo que recibió del movimiento sindical (Borja, 1975). Las asociaciones crearon redes de trabajo, intercambiándose preocupaciones, acciones y ayuda. La interacción al interior del barrio y entre barrios fue lo que permitió a las asociaciones su implicación con la estructura social más amplia. Al menos una tercera parte de las acciones de las asociaciones de vecinos estaba orientada a la escala de ciudad y región (Gail, 1979, p. 41).

Con el retorno de la democracia en España, el movimiento vecinal comenzó un progresivo proceso de institucionalización (Villasante, 2000). Muchos de los cuadros políticos y técnicos que lo acompañaron pasaron a formar parte de las administraciones locales y regionales, y desde allí impulsaron una 'regulación' de la acción vecinal, que tuvo como uno de sus principios 'devolverla' al ámbito barrial comunitario.

La cronología que propone Bonet i Martí (2012) indica que hasta 1979 se asistió al auge del movimiento vecinal, a la reivindicación de equipamientos y urbanización y a la oposición a las grandes operaciones urbanísticas con fines especulativos. De 1979 a 1983 se extiende el periodo de los consensos entre las nuevas autoridades y las asociaciones vecinales. Entre 1983 y 1990 se produjo la regulación de la participación ciudadana y la descentralización en distritos. La primera normativa en esta materia, en la década de los ochenta, dio naturaleza legal a la participación e introdujo, al mismo tiempo, cierta burocratización de la iniciativa ciudadana, que dificultó la introducción de componentes deliberativos y la implicación de la ciudadanía en los asuntos públicos (Villasante \& Gutiérrez, 2000). Por su parte, la descentralización en distritos aumentó las competencias administrativas de dichos territorios (Borja, 2001). En Barcelona, esta reorganización se produjo en 1984. Entre 1990 y 2000 se iniciaron los primeros Planes Comunitarios, y con ello el énfasis comenzó a ponerse más en el fortalecimiento del espacio comunitario barrial que en su relación con las políticas públicas y la producción de la ciudad. Desde 2000, la acción política municipal se ha venido focalizando en la intervención en los barrios con mayores carencias y en diseñar procesos de racionalización de la participación ciudadana. Luego, a través de la aprobación de la Ley 2/2004, del gobierno de la Generalitat se definió un marco para actuaciones focalizadas en barrios degradados de villas y áreas urbanas que requieren atención especial. En 2008, la Administración hizo una apuesta decidida por vincular los procesos de participación ciudadana con la descentralización en barrios, aprobando para ello la medida de gobierno "Els Barris de Barcelona". Esta medida institucionalizó la división municipal en 73 barrios, creando un nuevo espacio de participación: el Consejo de Barrio (Bonet i Martí, 2012). Finalmente, y a partir de la administración de Ada Colau, de corte progresista, al frente del gobierno de la ciudad desde 2015, se instituyó el Plan de Barrios en Barcelona que "pone en marcha acciones sociales, económicas y urbanas para mejorar los 
barrios que más lo necesitan" (Ayuntamiento de Barcelona, 2017a).

El proceso dominante de transformación de la relación entre lo vecinal y la producción de lo urbano en Barcelona ha sido el de institucionalización; es decir, el paso desde una participación por irrupción, que problematiza la noción de interés general y establece puentes entre lo particular y universal (la parte y la totalidad), hacia una participación por invitación, iniciativa de los poderes públicos y estructurada en órganos, mecanismos y procesos participativos (Bonet i Martí, 2012). Para Leiva, Miró y Urbano (2007), se trataba de una expropiación de la política al construir una arquitectura de representación profundamente institucionalizada, que ejercía el control de los conflictos y determinaba la gestión urbana:

(...) el cuerpo social se integra al Estado, pero la parte activa y responsable es únicamente la parte minoría dirigente. Todo intento de democracia directa, de organización del control del poder desde la base es invalidado y anulado de manera sistémica. (p. 65)

La institucionalización de la relación entre lo vecinal y la ciudad ha tenido crecientemente la escala barrial como protagonista. El fuerte movimiento vecinal de los setenta, articulado y con posicionamiento sobre la ciudad, poco a poco fue contenido espacialmente. Se desenvolvió en distritos y en barrios, se manifestó en agendas y demandas comunitarias acotadas a problemas de escala local, y se lo reguló a partir de un conjunto de normativas de participación también local.

Pese a lo anterior, es necesario hacer notar que aún existe en la esfera vecinal un potencial de articulación y politización que se continúa expresando en ciertas coyunturas y territorios. Ejemplo de ello son el rol que tuvo el movimiento vecinal en el $15 \mathrm{M}$ y en la conformación de los Comités de Defensa del Referéndum y de la República (CDR) en el marco del proceso independentista catalán; y las articulaciones territoriales que sobrepasan los límites del barrio y de sus agendas

\footnotetext{
${ }^{1}$ Cabe destacar que todos los valores que se enmarcan entre $63 \%$ y $79 \%$ se clasifican como rentas bajas; y aquellos que se encuentran por debajo de $63 \%$, como rentas muy bajas.
}

internas para actuar en una escala mayor, tal como ocurre en Nou Barris.

\section{El caso Nou Barris}

Antecedentes sociodemográficos. Nou Barris es el octavo distrito de los diez que conforman la ciudad de Barcelona. Con la aprobación de la actual división municipal de Barcelona en 1984, fue reconocido como un territorio más de la ciudad (Figura 1). Tiene una población de 164.881 personas, una cifra que lo sitúa como el quinto distrito más poblado y cuyos habitantes representan el 10,3\% de la población total de la ciudad (Ayuntamiento de Barcelona, 2018b). Está formado por trece barrios: Vilapicina i la Torre Llobeta, Porta, el Turó de la Peira, Can Peguera, la Guineueta, Canyelles, les Roquetes, Verdun, la Prosperitat, la Trinitat Nova, Torre Baró, Ciutat Meridiana y Vallbona.

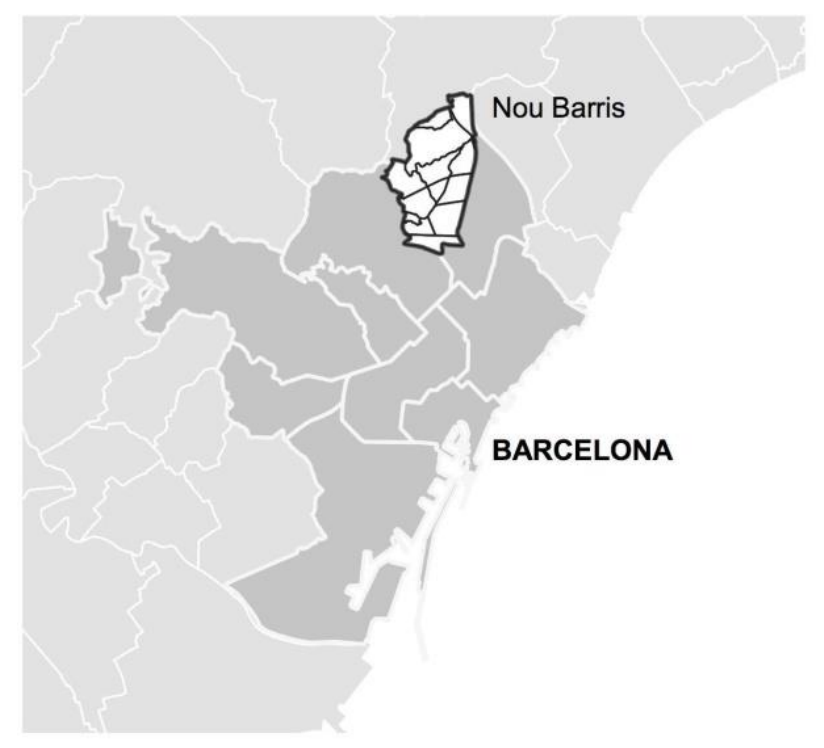

Figura 1. Distrito Nou Barris en Barcelona. Elaboración propia.

Nou Barris alberga ocho de los diez barrios más pobres de la ciudad. Es el distrito con la Renta Familiar Disponible per cápita (RFD) más baja de Barcelona, con un 53,8\% de la RFD,1 muy por debajo de la media de la ciudad, que se 
representa con el valor 100 (Ayuntamiento de Barcelona, 2017b). Mientras la tasa media de paro de Barcelona se situaba el año 2015 en un 7,7\%, en Nou Barris superaba el $14 \%$ (periodo en el cual el Distrito se moviliza). El 5,3\% de sus habitantes nunca ha ido a la escuela y solo el $13,8 \%$ ha ido a la universidad, un porcentaje mucho más bajo que la media de la ciudad, que se sitúa en un 30\% (Ayuntamiento de Barcelona, 2017). Desde los años 2000, con la llegada de personas procedentes de países de Latinoamérica, África o Asia, su población migrante pasó de 4.961 personas en 2001 a 27.100 en 2010.

El proceso de articulación vecinal de Nou Barris. Como producto de una política de valorización de las áreas centrales, se produjo entre los años 1950 y 1970 una masiva erradicación de moradores que vivían en edificios y casas precarias -o incluso en barracas - en el centro histórico de Barcelona o cerca de él. Parte de la población fue transferida a áreas muy distantes, sin ninguna infraestructura urbana, en las cuales se aceleró la construcción de polígonos habitacionales (Valdosky, 2017). En este contexto, Nou Barris experimentó un enorme crecimiento, que combinó vivienda pública de bajo costo, sectores de autoconstrucción y urbanizaciones privadas. Los dos primeros generalmente de baja calidad, sin servicios básicos, y con mínima infraestructura de conexión con el resto de la ciudad (Ayuntamiento de Barcelona, 2018a; Borja, 1975; Domingo i Clota \& Bonet i Casas, 1998, p. 40).

La necesidad de superar la precariedad urbana y el descontento político con el régimen franquista se constituyeron en un terreno fértil para el trabajo colaborativo entre vecinos, cuadros políticos y profesionales de izquierda (Andreu, 2015).

¿Qué nos cohesionó en aquel momento?, el ver que la gente llegaba aquí y que las criaturas tenían que ir a la escuela y no teníamos escuelas. Te ponías enferma y tenías que ir al médico, entonces necesitabas un CAP [Centro de Atención Primaria de salud]. Eso cohesionó. La lucha. Cuando yo llegué la calle mía estaba sin asfaltar. Por eso es importante la lucha por los derechos sociales, porque es lo que nos puede cohesionar. (Dirigenta Red Nou Barris Acull)

En este contexto, el ayuntamiento planteó el Plan Parcial Torre Baró-Valbona-Trintat, que implicaba la intervención de 535 hectáreas y el derribo de 4.370 viviendas. El plan daba prioridad a grandes vías de circulación y a la creación de espacios libres, para lo cual las inmobiliarias dispondrían de nuevos terrenos donde construir pisos, todo ligado con los intereses municipales (Valdosky, 2017). Con el fin de defenderse de este Plan, en abril de 1970 se creó la Asociación de Vecinos de Nou Barris, formada por nueve barriadas o secciones: Prosperitat, Verdum, Roquetes, Trinitat Vella, Trinitat Nova, Torre Baró, Vallbona, Guineueta y Ciutat Meridiana (Andreu, 2015, p. 52). De este modo, Nou Barris existió primero como espacio de resistencia y coordinación de organizaciones vecinales y luego, catorce años después, en 1984, como distrito. Su identidad fundacional no dependió de una decisión administrativa, sino de una práctica asociativa.

Hay dos formas de ver esto [la división de la ciudad en zonas]. Una es que administrativamente el Ayuntamiento divide la ciudad en distritos; y otra que es histórica, que es el caso de Nou Barris, que es bastante más excepcional. (Líder vecinal Nou Barris)

Yo creo que existe una identidad de Nou Barris. Mucha gente dice "tú ¿dónde vives?". "En Nou Barris". "¿Y en qué barrio?”, "En la Guinaueta, en Roquetas...", donde sea, pero sí que existe una identidad de Nou Barris. (Líder vecinal Nou Barris)

El proceso de constitución de Nou Barris, en tanto procedimiento de articulación vecinal, se fue fortaleciendo con la incorporación de más entidades y asociaciones. Durante los años setenta y ochenta se llevó a cabo un sinnúmero de acciones para reivindicar mejoras tanto en el acceso a la vivienda como en el espacio público, en el equipamiento deportivo y cultural, la sanidad, el transporte público y la conectividad con el resto de la ciudad, entre otras muchas condiciones. Un hito de este proceso fue la paralización y recuperación de una planta asfáltica y su transformación en el Ateneo Nou Barris en el año 1977 (Ayuntamiento de Barcelona, 2018a; Sasa, 2013).

La capacidad de acción colectiva desplegada en el episodio fundacional de articulación, que se inicia con la resistencia al Plan Parcial Torre Baró-Valbona-Trintat, permitió a la Asociación Nou Barris negociar numerosos adelantos urbanos con los primeros ayuntamientos 
democráticos: equipamientos para infantes, para jóvenes y adultos mayores; centros cívicos y culturales, bibliotecas, mercados municipales, centros de atención primaria de servicios sociales, instalaciones deportivas municipales, centros de salud y escuelas primarias, secundarias y para adultos, entre otros (Sasa, 2013).

Los años setenta y ochenta fueron un período de reivindicación pura y dura, porque en Nou Barris no había de nada a nivel de servicios básicos. En los años noventa se empezaron a conseguir equipamientos: hospitales, escuelas, centros culturales, "casals de joves", etcétera. Fue un proceso que permitió encarar los primeros años de los 2000 con unos cuantos objetivos alcanzados en cuanto a equipamientos básicos se refiere. Esto coincidió a nivel municipal con el mandato del llamado "tripartito" [Partit dels Socialistes de Catalunya (PSC), Esquerra Republicana de Catalunya (ERC) e Iniciativa per Catalunya Verds (ICV)]. Ese gobierno hizo mucho a nivel físico por los barrios. (Dirigenta, distrito Nou Barris)

Entre mediados de 1990 y buena parte de los 2000, en una situación económica nacional positiva, habiendo conseguido un enorme conjunto de adelantos urbanos y con una administración municipal abierta a las reivindicaciones vecinales, la movilización social fue disminuyendo y las entidades de cada barrio empezaron a mirar un poco más sus realidades particulares (Cano, 2017). Se mantuvo cierto nivel de coordinación entre las asociaciones vecinales, pero ello no se tradujo en una acción conjunta significativa.

La gente tenía trabajo (no había tanta penuria económica), se había conseguido una serie de equipamientos y la Administración la regentaba... un gobierno que atendía las reivindicaciones vecinales. La suma de esto provoca que la movilización social disminuyera. $Y$ como la movilización disminuye a nivel global, las entidades de cada barrio empiezan a mirarse un poco más su propio ombligo. Esto no quiere decir que no se trabaje en red, pues la Coordinadora seguía reuniéndose una vez al mes. Pero como desde el Ayuntamiento también había más facilidades de interlocución, cada entidad iba para concretar lo suyo, aunque lo suyo no quiere decir que sea en detrimento del que está al lado; pero no deja de ser solo suyo, es decir, de ese barrio. (Dirigenta distrito Nou Barris)

Donde el trabajo en red a nivel del distrito continuó siendo el eje principal fue en las plataformas enfocadas en temas específicos, que siguieron desarrollando un trabajo en red a nivel distrital. Entre ellas destacan la red Nou Barris Acull, creada en 1990 para facilitar la incorporación en los barrios de las personas provenientes de la inmigración; la Trobada Alternativa, un espacio de reflexión y acción política; y la plataforma 500×20, que se creó para luchar por un alquiler público asequible.

A partir de fines de los años 2000, la creciente evidencia de problemas socioeconómicos en el distrito, por efectos de la crisis de 2008 y de los sistemáticos recortes de las partidas públicas, puso en alerta las redes vecinales de Nou Barris, al tiempo que el $15 \mathrm{M}$ y el Movimiento de los Indignados sirvieron de impulso para que se activara nuevamente el trabajo articulado en el territorio, al que se sumaron nuevas plataformas: en 2013, la plataforma "Salvem les Pensions 9 Barris" y en 2014 la plataforma Aturats, que había surgido como producto del incremento de la tasa de paro en Nou Barris.

Durante un tiempo los grandes temas sociales estuvieron solucionados, pero cuando estos grandes temas dejaron de estar solucionados porque empezaron los recortes a todos los niveles y la pérdida de derechos, fue muy fácil trabajar de nuevo colectivamente, en red. No hubo ninguna dificultad. Es mucho más efectivo ponerse delante de la administración pública en bloque y con un montón de entidades detrás, que si lo hace una decena de personas de forma individual. (Dirigenta, Ateneu Popular Nou Barris)

En este contexto, en 2012, más de un centenar de asociaciones, entidades y redes lanzaron la campaña Nou Barris Cabrejada diu Prou! (Pauné, 9 y 10 de noviembre de 2012) y en noviembre de 2014 se publicó el informe "No es pobreza, es injusticia" (Nou Barris Cabrejada diu Prou!, 2014), que había comenzado a ser elaborado en 2012 a partir de un enorme esfuerzo de organización vecinal (Baquero, 2014). En el documento se observa el paso de una conciencia centrada en el concepto de pobreza a una orientada por el de injusticia: 
Tenemos derechos y no queremos caridad porque no es pobreza, es injusticia. (...) Porque no se trata de pobreza, sino de injusticia. En nuestra ciudad, como en la sociedad en general, están creciendo de manera exponencial las desigualdades sociales. Desigualdades entre rentas y territorios. En paralelo, los cortes brutales en los servicios públicos y derechos sociales, sumados a la falta de inversión en los barrios, agravan una situación de la que, además, culpabilizan a la población. (Nou Barris Cabrejada diu Prou!, 2014)

En el mismo año, la plataforma se movilizó para reclamar a los representantes políticos acciones concretas. Centenares de vecinos recorrieron las calles más céntricas de Barcelona hasta llegar a las puertas del Ayuntamiento y de la Generalitat. Una vez allí, leyeron un manifiesto donde se sostenía que lo que muchos barceloneses sufren no es pobreza, sino injusticia. La campaña Nou Barris Cabrejada solicitó al gobierno del distrito la realización de un pleno extraordinario en junio de 2016, del cual nació la medida de gobierno "Pla d'Acció per la Cohesió i els Drets Socials de Nou Barris 2016-2019" (Ayuntamiento de Barcelona, 2016; Guerrero, 2016), que contiene compromisos de gestión e inversión en diversas áreas, y que ha sido la base para el diseño de otras medidas de gobierno que consolidan, en el caso de Nou Barris, un enfoque que fortalece el distrito como escala de actuación.

Es de las plataformas más interesantes que se han hecho. También queda mucho recorrido por hacer y también se han de pulir muchas cosas, pero Nou Barris Cabreada tiene una referencialidad dentro del distrito y está siendo un polo, está generando un contradiscurso a las instituciones de tú a tú. (Líder social, Trovada alternativa, Nou Barris)

A tres años de este nuevo gran episodio de articulación vecinal, la evaluación que hacen los líderes de Nou Barris respecto de la implementación de las medidas negociadas con el Ayuntamiento de Barcelona y la Generalitat de Cataluña es diversa. Se reconoce que la campaña logró poner a Nou Barris en el centro de las políticas de la ciudad y que se ha avanzado en distintas áreas. Pero hay muchos aspectos donde los compromisos no se han cumplido (López, 2019). Quizá esta sea la razón de que la enorme votación que obtuvo en 2015 el partido de la alcaldesa Ada
Colau, Barcelona en Comù, no se repitiera ni en las elecciones parlamentarias, ni en municipales de 2019.

\section{Conclusiones}

En los últimos cuarenta años, el movimiento vecinal ha vivido en España un proceso creciente de fragmentación y atomización. Su capacidad de actuación se ha institucionalizado y ha quedado recluida en gran media en la escala del barrio; no del vivido, sino del concebido por las políticas urbanas: una geografía contenida de la acción vecinal. Lo vecinal urbano ha disminuido su capacidad para producir vínculo entre el habitar y lo político. $\mathrm{Ha}$ adoptado una espacialidad y una forma fragmentada y despolitizada. Se trata de una representación de la vida social urbana que ya no hace referencia a una particular forma de asentamiento humano (como los barrios que describen Certeau, Giard y Mayol, 1999), sino a un encapsulamiento espacial y político de la vida en la ciudad.

En este contexto, es interesante observar el caso de Nou Barris. Su red de trabajo asociativo, fundada a principios de 1970, mucho antes de constituirse como distrito, no solo ha resistido el impacto de las políticas que ponen el foco en el barrio, sino que ha venido complejizándose a partir de sucesivas luchas sociales y de la constitución y agregación de numerosas redes y plataformas. Si bien a mediados de los noventa la articulación asociativa en Nou Barris se debilitó, y la acción vecinal se contuvo en los barrios, la crisis de 2008 activó una geografía vecinal más articulada, presente en la memoria y las redes de trabajo asociativo fundadas y consolidadas en los años setenta y ochenta.

La espacialidad y forma articulada de acción vecinal de las organizaciones de Nou Barris les permite actuar a escala de distrito, lo que les otorga una voz política significativa para dialogar con la autoridad (Bonet i Martí, 2012; Borja, 1975; Jacobs, 2011). Su acción vecinal tiene mecanismos para traducir los problemas cotidianos a la esfera pública. Estos mecanismos trabajan en dos ejes: uno que articula el espacio cotidiano (la calle y el barrio) con el territorio mayor (distrito) y que está conformado por las organizaciones de cada barrio y la Coordinadora de asociaciones y entidades Nou Barris; y otro que articula los problemas cotidianos (el desempleo, la migración, las pensiones) con sus contextos sociopolíticos mayores. Así, las diversas plataformas de Nou Barris contribuyen a politizar el habitar vecinal, reivindicando las 
desigualdades socioespaciales y vinculándolas no solo con el contexto local, sino también con los procesos globales, porque tanto la dimensión local como la global tienen efectos sobre la manera en que se construye el orden social de un vecindario (Massey, 2012). La acción articulada de estos dos ejes, el territorial y el temático, ha logrado tensionar y movilizar la actuación de las políticas púbicas, relevando el distrito como escala en la cual el ayuntamiento y el gobierno de Cataluña deben actuar.

Si bien los barrios, como espacios vividos, han cumplido y siguen cumpliendo un papel relevante en Nou Barris, lo distintivo es la capacidad que han tenido sus líderes y dirigentes para construir vínculos más allá de ellos. Sin negar la pertenencia a cada barrio, se han tejido relaciones de carácter más estratégico en una escala complementaria, que está en relación con los propósitos y las agendas que las organizaciones vecinales se plantean (Madden, 2014; Suttles, 1972).
El caso de Nou Barris supera la noción tradicional de barrio y su modo de concebir las relaciones vecinales. Su geografía de la acción vecinal es una forma de resistencia a la hegemonía que la abstracción de barrio impone al tejido asociativo territorial. Evoca la actividad creadora, niega la fragmentación y despolitización del espacio. La dialéctica que se produce entre barrio, distrito y ciudad es una condición que facilita la participación vecinal en la construcción de lo urbano. La articulación de espacialidades diversas, a partir de la construcción de redes vecinales entre organizaciones ancladas en distintos lugares, propicia el surgimiento de escalas de actuación con mayor capacidad de politización del habitar y de actuar como interlocutores de la autoridad. Es la apropiación de lo urbano, la reivindicación de su valor de uso y su condición de obra producida por los propios sujetos (Lefebvre, 1969, 1974) [B]

\section{Referencias}

Andersson, R. \& Musterd, S. (2005). Area-based policies: A critical appraisal. Tijdschrift Voor Economische En Sociale Geografie, 96(4), 377-89. https://doi.org/10.1111/j.1467-9663.2005.00470.x

Andreu, M. (2015). Barris, veïns i democràcia: El moviment ciutadà i la reconstrucció de Barcelona (1968-1986). Barcelona: L'Avenç.

Atkinson, R., Dowling, R., \& McGuirk, P. (2009). Home/Neighbourhood/City/+. Environment and Planning, 41(12), 2816-22. Recuperado de https://doi.org/10.1068\%2Fa42110

Ayuntamiento de Barcelona (2016). Mesura de Govern. Pla d'Acció per la Cohesió i els Drets Socials de Nou Barris 2016-2019. Consell del Districte de Nou Barris. Sessió extraordinària 19 de maig de 2016.

Recuperado de

http://ajuntament.barcelona.cat/premsa/wpcontent/uploads/2016/05/mesuragovernnoubarris.p $\underline{\mathrm{df}}$
Ayuntamiento de Barcelona (2017a). Pla de barris, Barcelona (2016-2020) / El plan de los barrios de Barcelona. Recuperado de https://pladebarris.barcelona/es

Ayuntamiento de Barcelona (2017b). Distribució territorial de la renda familiar disponible per càpita a Barcelona. (Gabinet Tècnic de Programació. Departament d'Estudis i Programació). Recuperado de www.barcelona.cat. https://bit.ly/2Kw7Qyc

Ayuntamiento de Barcelona. (2018a). Breve historia de Nou Barris. Recuperado de https://ajuntament.barcelona.cat/noubarris/es/cono zca-el-distrito/historia

Ayuntamiento de Barcelona. (2018b). Guía de estadísticas. Nou Barris en cifras. Recuperado de http://www.bcn.cat/estadistica/catala/documents/di strictes/08 NouBarris 2018.pdf 
Ayuntamiento de Barcelona (2017). Guía de estadísticas. Nivel académico del distrito Nou Barris, 2017. Recuperado de https://www.bcn.cat/estadistica/castella/dades/guia dt08/pob08/t19.htm

Baquero, C. (20 de noviembre de 2014). El memorial de agravios de Nou Barris. El País. Recuperados de https://elpais.com/ccaa/2014/11/20/catalunya/1416 511908 286112.html

Bettin, G. (1982). Los sociologos de la ciudad. Barcelona: Gustavo Gili.

Bonet i Martí, J. (2012). El territorio como espacio de radicalización democrática. Una aproximación crítica a los procesos de participación ciudadana en las políticas urbanas de Madrid y Barcelona. Athenea DigitalK, 12(1), 15-28. Recuperado de https://dialnet.unirioja.es/servlet/articulo?codigo $=41$ 49752

Borja, J. (1975). Movimientos sociales urbanos. Buenos Aires: Siap-Planteos.

Borja, J. (2001). Ciutadans i participació. Participació ciutadana. Ponencia presentada en el 20 Congreso de Municipios de Catalunya, Barcelona, España.

Brenner, N. (2004). Urban governance and the production of new state spaces in western Europe, 1960-2000. Review of International Political Economy, 11(3), 447-488. https://doi.org/10.1080/0969229042000282864

Brenner, N. \& Theodore, N. (2002). Cities and the geographies of "actually existing neoliberalism." Antipode, 34(3), 349-79. https://doi.org/10.1111/1467-8330.00246

Cano, B. (2017). Malestar social y tiempos de populismo: crisis y desafección política en Nou Barris. Trabajo de fin de grado, Departament de Mitjans, Comunicació i Cultura, Universidad Autónoma de Barcelona.

Certeau, M. de, Giard, L., \& Mayol, P. (1999). La invención de lo cotidiano. 2. Habitar, cocinar. México, DF: Universidad Iberoamericana; Instituto Tecnológico de Estudios Superiores de Occidente.
Davidson, M. (2008). Spoiled mixture: Where does stateled 'positive' gentrification end? Urban Studies, 45(12), 2385-2405. https://doi.org/10.1177\%2F0042098008097105

Domingo i Clota, M. \& Bonet i Casas, R. M. (1998). Barcelona i els moviments socials urbans. Barcelona: Mediterrània. Recuperado de https://www.fbofill.cat/sites/default/files/1034.pdf

Drake, P.W. \& Jaksic, I. (1999). El modelo chileno: democracia y desarrollo en los noventa. Santiago de Chile: Lom.

Espinoza, V. (1998). Historia social de la acción colectiva urbana: Los pobladores de Santiago, 1957-1987. EURE, 24(72), 71-84. https://doi.org/10.4067/S0250$\underline{71611998007200004}$

Gail Bier, A. (1979). "Vox populi": El desarrollo de las asociaciones de vecinos en España. Papers. Revista de Sociología, (11), 169-183. http://dx.doi.org/10.5565/rev/papers/v11n0.1164

Galster, G., Andersson, R., \& Musterd, S. (2010). Who is affected by neighbourhood income mix? Gender, age, family, employment and income differences. Urban Studies, 47(14), 2915-44. https://doi.org/10.1177\%2F0042098009360233

Garnier, J.-P. (2011). Del derecho a la vivienda al derecho a la ciudad: ¿De qué derechos hablamos... y con qué derecho? Biblio 3W. Revista Bibliográfica de Geografía y Ciencias Sociales, 16(909). http://www.ub.edu/geocrit/b3w-909.htm

Giddens, A. (1993). Consecuencias de la Modernidad. Traducido por A. Lizón Ramón. Madrid: Alianza Universidad.

Granovetter, M. S. (1973). La fuerza de los vínculos débiles. American Journal of Sociology, 78(6), 136080. Recuperado de https://dialnet.unirioja.es/servlet/articulo?codigo $=15$ $\underline{4588}$

Harvey, D. (1997). The new urbanism and the communitarian trap. Harvard Design Magazine (1). http://www.harvarddesignmagazine.org/issues/1/the -new-urbanism-and-the-communitarian-trap 
Harvey, D. (2012). La geografía como oportunidad política de resistencia y construcción de alternativas. Revista de Geografía Espacios, 2(4), 9-26. http://dx.doi.org/10.25074/07197209.4.339

Jacobs, J. (2011 [1961]). Muerte y vida de las grandes ciudades. Barcelona: Capitan Swing.

Keller, S. (1979). El vecindario urbano, una perspectiva sociológica (2a ed.). Madrid: Siglo XXI.

Guerrero, D. (17 de mayo de 2016). Inversión de 40 millones en Nou Barris. La Vanguardia. https://www.lavanguardia.com/local/barcelona/2016 0517/401847820058/inversion-nou-barris.html

Lefebvre, H. (1969). El derecho a la ciudad. Barcelona: Península.

Lefebvre, H. (1971). De lo rural a lo urbano. Barcelona: Península.

Lefebvre, H. (2013 [1974]). La producción del espacio. Barcelona: Capitán Swing.

Leiva, E., Miró, I., y Urbano, X. (2007). De la protesta al contra poder: nous protagonismes socials en la Barcelona metropolitana. Barcelona: Virus.

Letelier, L. (2018). El barrio en cuestión. Fragmentación y despolitización de lo vecinal en la era neoliberal. Scripta Nova, 22. https://doi.org/10.1344/sn2018.22.21518

Letelier, F., Tapia, V. y Boyco, P. (2018). ¿Nuevas territorialidades vecinales en el Chile neoliberal? Polis, 17(49), 55-78. http://dx.doi.org/10.4067/S0718$\underline{65682018000100055}$

Lopes de Souza, M. (2016). Lessons from praxis: Autonomy and spatiality in contemporary Latin American Social Movements. Antipode, 48(5), 12921316. https://doi.org/10.1111/anti.12210

López, H. (13 de mayo de 2019). Chequeo electoral: Nou Barris se siente "más escuchado", pero pide más resultados. El Periódico. Recuperado de https://www.elperiodico.com/es/barcelona/2019051 3/nou-barris-sigue-cabreado-7435424
Madden, D. J. (2014). Neighborhood as spatial project: Making the urban order on the downtown Brooklyn waterfront. International Journal of Urban and Regional Research, 38(2), 471-497. https://doi.org/10.1111/1468-2427.12068

Martin, D. (2003). Enacting neighborhood. Urban Geography, 24(5), 361-385. https://doi.org/10.2747/0272-3638.24.5.361

Martínez, E. (1999). Introducción. En R. E. Park (ed.), La ciudad y otros ensayos de ecología urbana. Barcelona: Ediciones del Serbal.

Massey, D. (2007). Geometrías del poder y la conceptualización del espacio. Conferencia dictada en la Universidad Central de Venezuela, Caracas, 17 de setiembre, 2007.

Massey, D. (2012). Un sentido global del lugar. En A. Albet y N. Benachy N. (eds.), Doreen Massey. Un sentido global del lugal, pp. 112-228. Barcelona: Icaria.

Merrifield, A. (2011). El derecho a la ciudad y más allá: notas sobre una reconceptualización lefebvriana. Urban, 2, 101-110. Recuperado de https://dialnet.unirioja.es/servlet/articulo?codigo $=37$ $\underline{62685}$

Mesa, A. (24 de marzo de 2017). El movimiento vecinal de Barcelona: una historia de vuelta a empezar. Metrópoli abierta, Barcelona. Recuperado de http://www.metropoliabierta.com/opinion/elmovimiento-vecinal-de-barcelona-una-historiade 757 102.html

Nou Barris Cabrejada diu prou! | Barcelona (2014). No és pobresa, és injusticia. Recuperado de https://jaumeuab.files.wordpress.com/2015/01/docu mentpobresa definitiu-1.pdf

Panfichi, A. (1996). Del vecindario a las redes sociales: cambio de perspectivas en la sociología urbana. Debates en Sociología, (20-21). http://revistas.pucp.edu.pe/index.php/debatesensoci ologia/article/view/6949

Park, R. E., Burgess, E. W., \& McKenzie, R. (1925). The City. Chicago: University of Chicago Press. 
Park, Y. \& Rogers, G. O. (2014). Neighborhood planning theory, guidelines, and research: Can area, population, and boundary guide conceptual framing? Journal of Planning Literature, 30(1), 18-36. https://doi.org/10.1177\%2F0885412214549422

Pauné, M. M. (9 de noviembre de 2012). El 'cabreo' de Nou Barris baja a la calle, por M. M. Pauné. $L a$ Vanguardia . Recuperado de https://www.lavanguardia.com/local/barcelona/2012 1109/54354944329/cabreo-nou-barris-calle.html

Pauné, M. M. (10 de noviembre de 2012). Nou Barris está 'cabreado'. La Vanguardia . Recuperado de https://www.lavanguardia.com/local/barcelona/2012 1010/54352429369/nou-barris-cabreado.html

Perry, C. A. (2007 [1929]). The neighborhood unit. En M. Larice and E. Macdonald (eds.), The Urban Design Reader, pp. 54-65. Londres: Routledge.

Purcell, M. (2014). Possible worlds: Henri Lefebvre and the right to the city. Journal of Urban Affairs, 36(1), 141-54. https://doi.org/10.1111/juaf.12034

Raffestin, C. \& Butler, S. A. (2012). Space, territory, and territoriality. Environment and Planning D: Society and Space, 30(1), 121-141. https://doi.org/10.1068\%2Fd21311

Rodríguez, G. (2008). ¿Comunidad? Mediación comunitaria, habitar efímero y diversidad cultural. Polis, 7(20). http://dx.doi.org/10.4067/S0718$\underline{65682008000100006}$

Sanz, L. (2003), Análisis de redes sociales: o cómo representar las estructuras sociales subyacentes. Consejo Superior de Investigaciones Científicas, Unidad de Políticas Comparadas. Documento de Trabajo 03-07, 20-29.

http://digital.csic.es/bitstream/10261/1569/1/dt0307.pdf

Sasa, Z. (2013). El modelo Barcelona de Espacio Público y Diseño Urbano: Consolidación urbana de Nou barris a través de la red de espacios públicos [Tesis]. Master en Diseño Urbano: Arte, Ciudad, Sociedad, Facultad de Bellas Artes, Universidad de Barcelona. http://hdl.handle.net/2445/33303

Sevilla, A. (2017). Neil Brenner. Teoría urbana crítica y políticas de escala. Barcelona: Icaria-Espacios Críticos.
Silver, C. (1985), Neighborhood planning in historical perspective. Journal of the American Planning Association, 51(2), 161-174. https://doi.org/10.1080/01944368508976207

Suttles, G. (1972), The social contruction of communities. Chicago: University of Chicago Press.

Tapia Barría, V. (2018). Geografías de la contención: el rol de las políticas de escala barrial en el Chile neoliberal Scripta Nova, 22(592). http://revistes.ub.edu/index.php/ScriptaNova/article /view/20272

Tapia, V. (2018). Geografías de la contención: el rol de las políticas de escala barrial en el Chile neoliberal. Scripta Nova, 22(592).

http://revistes.ub.edu/index.php/\%20ScriptaNova/art icle/view/20272

Valdosky, F. (2017). Das ações de resistência à consciência da expropriação. En A. F. Alessandri Carlos, G. Alves y R. Faleiros de Padua (orgs.), Justiça Espacial e o direito a cidade. São Paulo: Editora Contexto.

Villasante, T. (1999). Redes y socio-praxis. Politica y Sociedad, 1(23). https://webs.ucm.es/info/pecar/Articulos/Villasante2 1.pdf

Villasante, T. \& Gutiérrez V. (2000). El movimiento vecinal: trayectoria y perspectivas. En E. Grau y P. Ibarra Güell (coords.), Anuario de Movimientos Sociales 2000. Participando en la Red, pp. 70-86. Barcelona: Icaria - betiko Fundazioa. Recuperado de http://fundacionbetiko.org/wpcontent/uploads/2012/11/el-movimiento-vecinaltrayectoria-y-perspectivas.pdf

Wellman, B. (1979). The community question: The intimate networks of East Yorkers. American Journal of Sociology, 84(5), 1201-31. https://doi.org/10.1086/226906

Wellman, B. (2001). The persistence and transformation of community: From neighbourhood groups to social networks. 
Wellman, B. \& B. Leighton (1979). Networks, neighborhoods, and communities: Approaches to the study of community question. Urban Affairs

Quarterly, 14(3), 363-390.

https://doi.org/10.1177/107808747901400305
Young, I. M. (2000). La justicia y la política de la diferencia. Madrid: Universitat de Valencia, Instituto de la Mujer. 